\title{
Percepción de médicos de una universidad de Lima sobre su capacitación en procedimientos médicos durante el internado
}

Perception of physicians from a Medical school in Lima on training in medical procedures during internship

\author{
Álvaro Taype-Rondán ${ }^{1, a}$, Gustavo Tataje Rengifo, ${ }^{2, a}$, Angela Arizabal $^{2, a}$, \\ Saela Alegría Hernández ${ }^{2, b}$ \\ ${ }^{1}$ CRONICAS Centro de Excelencia en Enfermedades Crónicas, Universidad Peruana Cayetano Heredia, Lima, Perú. \\ ${ }^{2}$ Universidad de San Martín de Porres, Lima, Perú. \\ ${ }^{a}$ Médico Cirujano; ${ }^{b}$ Estudiante de Medicina Humana.
}

\begin{abstract}
Resumen
Objetivos. Evaluar la percepción de médicos recién egresados sobre su capacitación en procedimientos médicos durante el internado, y determinar los factores asociados a recibir una adecuada capacitación en un mayor número de procedimientos, en una universidad de Lima, Perú. Diseño. Estudio observacional, transversal y analítico. Institución. Universidad de San Martín de Porres, Lima, Perú. Participantes. Médicos egresados. Métodos. Estudio tipo encuesta realizado en médicos egresados de una universidad privada de Lima, Perú, que realizaron el internado médico durante el año 2014. Se utilizó la red social Facebook para ubicar a los participantes, se obtuvo el consentimiento informado y se envió una encuesta autoaplicada con variables sociodemográficas y referentes a su internado médico. Principales medidas de resultados. Percepción sobre su capacitación en procedimientos médicos durante el internado. Resultados. Se analizaron 117 encuestas. El número de horas laboradas por día fue en promedio 10,0 011,8, la frecuencia de carga laboral excesiva y de 'papeleo excesivo' en al menos una rotación fueron $80,3 \%$ y $84,6 \%$, respectivamente. Pocos encuestados recibieron durante su internado una adecuada capacitación en colocación de inyectables $(53,9 \%)$, reducción de fracturas $(28,2 \%)$ y atención de partos vaginales verticales $(27,4 \%)$. Haber percibido una adecuada capacitación en más de 20 procedimientos se asoció a considerar una adecuada docencia en tres o cuatro rotaciones ( $R P=2,55, I C 95 \%=1,36$ a 4,76) y laborar en promedio 9 horas y 30 minutos a 10 horas y 15 minutos diarios en comparación con laborar menos horas diarias $(R P=1,91, I C 95 \%=1,01$ a 3,64). Conclusiones. El exceso de carga laboral y el papeleo, asi como una jornada laboral reducida, están asociados a una menor cantidad de procedimientos médicos en los que el interno se perciba adecuadamente capacitado. Es necesario implementar estrategias para mejorar la docencia en el internado.
\end{abstract}

Palabras clave. Educación Médica; Formación de Recursos Humanos; Internado y Residencia.

\begin{abstract}
Objectives. To evaluate the perception of recently graduated physicians on their training in medical procedures during internship, and to determine factors associated to adequate training in a greater number of procedures at a university in Lima, Peru. Design. Observational, cross-sectional, analytical study. Setting. Universidad de San Martín de Porres, Lima, Peru. Participants. Recently graduated physicians. Methods. Survey study in medical graduates from a private university in Lima, Peru, who finished medical internship in 2014. Facebook social network was used to locate participants. Informed consent was obtained and a self-report survey was sent to collect sociodemographic characteristics and perceptions on their medical internship. Main outcome measures. Perception on medical procedures training during internship. Results. One hundred seventeen surveys were analyzed. The number of hours worked per day was on average $10.0 \pm 1.8$, the frequency of excessive workload and "excessive paperwork" in at least one rotation was respectively $80.3 \%$ and $84.6 \%$. Few respondents received adequate training during their internship in the administration of injections $(53.9 \%)$, reduction of fractures $(28.2 \%)$, and vertical vaginal deliveries $(27.4 \%)$. Having adequate training in more than 20 procedures was associated to having an adequate teaching in three or four rotations ( $P R=2.55,95 \% \mathrm{Cl}=1.36$ to 4.76$)$, and to work on average 9 hours 30 minutes to 10 hours 15 minutes daily compared with less working hours per day $(P R=1.91,95 \% \mathrm{Cl}=1.01$ to 3.64$)$. Conclusions. Excessive workload and paperwork as well as reduced working hours seem to affect negatively the number of medical procedures in which the interns feel adequately trained. It is necessary to implement strategies to improve teaching at boarding school. Keywords. Medical Education; Human Resources Formation; Internship and Residency.
\end{abstract}

An Fac med. 2016;77(1):31-8 / http://dx.doi.org/10.15381/anales.v77i1.11550

\section{INTRODUCCIÓN}

El internado médico es una etapa obligatoria en la formación de todo médico, en la cual los estudiantes participan de forma activa en los establecimientos de salud asignados, asumiendo responsabilidades sobre los pacientes que tienen a su cargo, participando activamente en las visitas médicas, realizando procedimientos médicos y consolidando los conocimientos adquiridos durante las etapas previas ${ }^{(1-3)}$. En Perú, el internado es realizado durante el último año del plan de estudios de pregrado, y suele comprender cuatro rotaciones ex- clusivamente hospitalarias: medicina, cirugía, pediatría y gineco-obstetricia.

Uno de los objetivos del internado médico en el Perú es capacitar a los internos en la realización práctica de procedimientos médicos básicos, que van a realizar como médicos generales. Esta es una preocupación especial en 
el Perú debido a que la mayoría de los médicos recién graduados va a realizar con prontitud el Servicio Rural y Urbano Marginal de Salud (SERUMS), durante el cual probablemente tengan que laborar en comunidades aisladas y realizar procedimientos médicos sin apoyo ${ }^{(4,5)}$.

Si bien la enseñanza teórica y simulada de estos procedimientos debería realizarse escalonadamente a lo largo de la carrera, es en el internado donde los estudiantes tienen la oportunidad de realizarlos repetidamente y con la supervisión adecuada, hasta consolidarlos ${ }^{\left({ }^{6}\right)}$. Sin embargo, se han reportado diversos problemas en el internado que podrían dificultar el aprendizaje y la realización de procedimientos por parte del interno, como sobrecarga laboral $(7,8)$, gran cantidad de trabajo administrativo (papeleo) ${ }^{(9,10)}$, inadecuada docencia, falta de supervisión de los procedimientos realizados ${ }^{(2)}$, falta de oportunidades para realizar ciertos procedimientos ${ }^{(11)}$, entre otros.

En Perú no se han publicado estudios que se centren en describir la enseñanza que se brinda en el internado médico. Esta falta de información impide posar una mirada crítica y elaborar estrategias para mejorar la formación médica. Por lo cual se realizó el presente estudio con el objetivo de evaluar la percepción de médicos recién egresados sobre su capacitación en procedimientos médicos durante el internado, así como determinar los factores asociados a recibir una adecuada capacitación en un mayor número de procedimientos, en una universidad privada de Lima, Perú.

\section{MÉTODOS}

Se realizó un estudio observacional, transversal y analítico en médicos egresados de la Universidad de San Martín de Porres (Lima, Perú), que hubieran realizado el internado médico en la ciudad de Lima durante el año 2014. En los currículos de dicha universidad no existen rotaciones de externado antes del internado.
Se utilizó el listado de internos de Medicina Humana de la universidad en cuestión que rindieron el Examen Nacional de Medicina durante el año 2014, publicado por la Asociación Peruana de Facultades de Medicina (ASPEFAM). Durante el periodo febrero a abril de 2015 se ubicó mediante la red social Facebook a cada una de las personas que figuraban en dicha lista y se realizó el consentimiento informado mediante el envío de un documento explicativo que incluía un consentimiento informado con la descripción del objetivo del estudio, datos del autor principal para contacto y detalles sobre el carácter voluntario y anónimo de la encuesta. A aquellos que aceptaron participar se les envió una encuesta diseñada para ser autoaplicada, solicitándoles que la llenen y la envíen por correo electrónico a una dirección creada ad hoc.

La encuesta incluyó las siguientes variables: sexo, edad, estado civil, sede hospitalaria, promedio de horas laboradas diariamente (sin contar las guardias nocturnas) y promedio de guardias realizadas mensualmente durante el internado. Además, se preguntó si la carga laboral fue excesiva, si el 'papeleo' ocupó la mayor parte de su tiempo, y si la docencia clínica fue adecuada. Estas tres preguntas tuvieron alternativas de tipo Likert (muy de acuerdo, de acuerdo, neutral, en desacuerdo, muy en desacuerdo). Posteriormente, las respuestas a estas preguntas fueron dicotomizadas, tomando como afirmativas las dos primeras alternativas (muy de acuerdo y de acuerdo), y como negativas el resto de alternativas.

También se recolectó la percepción sobre la capacitación recibida en procedimientos clínicos, quirúrgicos y gineco-obstétricos durante el internado. Para hallar el promedio del número de horas laboradas y del número de guardias, se calculó el promedio aritmético para las cuatro rotaciones. Para ello, elaboramos una lista de 29 procedimientos médicos básicos: 16 procedimientos clínicos, 7 procedimientos quirúrgicos y 6 procedimientos ginecoobstétricos. En un primer momento, se elaboró una lista de las competencias médicas recomendadas por la Comisión para la Acreditación de facultades o escuelas de Medicina Humana del Perú ${ }^{(12)}$, Scottish Deans' Medical Education Group (13), Tomorrow's Doctors ${ }^{(14)}$, Association of American Medical Colleges (15), el proyecto Tuning (16) y CanMEDS (17). Posteriormente, se revisó dicha lista para escoger los procedimientos más importantes para los médicos que van a realizar el SERUMS, según consenso entre los autores.

Las sedes hospitalarias fueron clasificadas de acuerdo a su grado de complejidad del I al IV, según la normativa vigente del Ministerio de Salud del Perú ${ }^{(18)}$. También fueron clasificadas de acuerdo a su pertenencia en: hospitales del Ministerio de Salud (MINSA), del Seguro Social (EsSalud), de las Sanidades (Fuerza Aérea del Perú, Policía Nacional del Perú, Ejército del Perú y Marina de Guerra del Perú), y Clínicas (hospitales privados).

Tabla 1. Datos generales de la población encuestada $(\mathrm{N}=117)$.

\begin{tabular}{|c|c|c|}
\hline & $N$ & $\%$ \\
\hline \multicolumn{3}{|l|}{ Sexo } \\
\hline Masculino & 51 & $43,6 \%$ \\
\hline Femenino & 66 & $56,4 \%$ \\
\hline \multicolumn{3}{|l|}{ Edad } \\
\hline 23 a 24 & 43 & $36,8 \%$ \\
\hline 25 a 26 & 61 & $52,1 \%$ \\
\hline 27 a 28 & 10 & $8,5 \%$ \\
\hline 29 a 30 & 3 & $2,6 \%$ \\
\hline \multicolumn{3}{|l|}{ Estado civil } \\
\hline Soltero & 116 & $99,1 \%$ \\
\hline Casado & 1 & $0,9 \%$ \\
\hline \multicolumn{3}{|l|}{ Sede hospitalaria } \\
\hline Más de un hospital & 15 & $12,8 \%$ \\
\hline MINSA & 13 & $11,1 \%$ \\
\hline EsSalud & 2 & $1,7 \%$ \\
\hline Un solo hospital & 102 & $87,2 \%$ \\
\hline Minsa & 66 & $56,4 \%$ \\
\hline EsSalud & 17 & $14,5 \%$ \\
\hline Sanidades & 13 & $11,1 \%$ \\
\hline Clínica & 6 & $5,2 \%$ \\
\hline
\end{tabular}


En cuanto a los aspectos éticos de la investigación, se respetó el carácter voluntario y anónimo de la encuesta. Asimismo, se vigiló la confidencialidad de los datos, que solo fueron manejados por los autores del estudio. El protocolo de investigación fue aprobado por el Comité de Ética del Hospital Nacional Docente Madre Niño San Bartolomé.

Una vez recibidas por correo electrónico, las encuestas fueron digitadas por uno de los autores en el programa Microsoft Excel 2010. A continuación, otro autor realizó una revisión de la calidad del $10 \%$ de las encuestas, sin encontrar errores. No se digitaron las direcciones de los correos electrónicos desde las cuales fueron enviadas las encuestas.
Para el análisis de los datos se utilizó el paquete estadístico STATA v13.0. Los resultados fueron presentados descriptivamente utilizando medidas de dispersión, de tendencia central y frecuencias absolutas y relativas. En tanto que el cruce de variables se realizó mediante la prueba de Kruskall Wallis y la prueba exacta de Fisher.

Para calcular los factores asociados a haber sido adecuadamente capacitados en un mayor número de procedimientos, se dicotomizó el número de procedimientos teniendo como punto de corte la división entre los dos primeros terciles y el tercer tercil. Se utilizó la regresión de Poisson simple y la regresión de Poisson múltiple con varianzas robustas. Se consideró significativo un $\mathrm{p}<0,05$.

\section{RESULTADOS}

La encuesta fue enviada a los 227 médicos de la universidad estudiada que realizaron el internado durante el año 2014, recibiendo respuesta de 119 , de las cuales dos fueron eliminadas por no tener todas las variables de interés completas. Finalmente se analizaron 117 encuestas $(51,5 \%$ del total), de los cuales $56,4 \%$ fueron de sexo femenino, $88,9 \%$ tuvieron entre 23 y 26 años, y $12,8 \%$ rotaron por más de un hospital durante su internado (tabla 1). Los encuestados rotaron en 24 sedes hospitalarias diferentes.

El número de horas laboradas por día sin contar las guardias nocturnas fue en promedio $10,0 \pm 1$, 8 horas, siendo significativamente mayor en se-

Tabla 2. Horas laboradas diariamente, guardias mensuales, percepción de carga laboral, papeleo y docencia según sedes hospitalarias en la población encuestada.

\begin{tabular}{|c|c|c|c|c|c|c|}
\hline & $\begin{array}{c}\text { Total } \\
(\mathrm{N}=117)\end{array}$ & $\begin{array}{l}\text { MINSA } \\
(\mathrm{N}=79)\end{array}$ & $\begin{array}{l}\text { EsSalud } \\
(\mathrm{N}=19)\end{array}$ & $\begin{array}{l}\text { Sanidades } \\
(N=13)\end{array}$ & $\begin{array}{l}\text { Clínicas } \\
(\mathrm{N}=6)\end{array}$ & $\mathrm{p}$ \\
\hline Horas laboradas & $10,0 \pm 1,8$ & $10,1 \pm 1,6$ & $10,6 \pm 2,3$ & $9,5 \pm 1,4$ & $8,1 \pm 1,7$ & $0,035^{*}$ \\
\hline Guardias por mes & $7,3 \pm 3,7$ & $7,9 \pm 4,3$ & $5,7 \pm 1,5$ & $6,3 \pm 1,7$ & $5,9 \pm 1,4$ & $0,014^{* *}$ \\
\hline \multicolumn{7}{|l|}{ La carga laboral fue excesiva } \\
\hline En al menos una rotación & $94(80,3)$ & $69(87,3)$ & $16(84,2)$ & $7(53,9)$ & $2(33,3)$ & 0,001 \\
\hline R. Medicina & $78(66,7)$ & $57(72,2)$ & $13(68,4)$ & $6(46,2)$ & $2(33,3)$ & 0,085 \\
\hline R. Gineco-Obs & $70(59,8)$ & $51(64,6)$ & $15(79,0)$ & $3(23,1)$ & $1(16,7)$ & 0,001 \\
\hline R. Cirugía & $50(42,7)$ & $35(44,3)$ & $7(36,8)$ & $6(46,2)$ & $2(33,3)$ & 0,890 \\
\hline R. Pediatría & $31(26,5)$ & $25(31,7)$ & $6(31,6)$ & $0(0,0)$ & $0(0,0)$ & 0,042 \\
\hline \multicolumn{7}{|c|}{ El 'papeleo' ocupó la mayor parte de su tiempo } \\
\hline En al menos una rotación & $99(84,6)$ & $72(91,1)$ & $17(89,5)$ & $7(53,9)$ & $3(50,0)$ & 0,001 \\
\hline R. Medicina & $89(76,1)$ & $65(82,3)$ & $14(73,7)$ & $7(53,9)$ & $3(50,0)$ & 0,058 \\
\hline R. Gineco-Obs & $74(63,3)$ & $56(70,9)$ & $13(68,4)$ & $2(15,4)$ & $3(50,0)$ & 0,001 \\
\hline R. Cirugía & $62(53,0)$ & $47(59,5)$ & $10(52,6)$ & $3(23,1)$ & $2(33,3)$ & 0,074 \\
\hline R. Pediatría & $53(45,3)$ & $45(57,0)$ & $4(21,1)$ & $1(7,7)$ & $3(50,0)$ & 0,001 \\
\hline \multicolumn{7}{|l|}{ La docencia clínica fue adecuada } \\
\hline En todas las rotaciones & $36(30,8)$ & $25(31,7)$ & $4(21,1)$ & $5(38,5)$ & $2(33,3)$ & 0,723 \\
\hline R. Medicina & $83(70,9)$ & $51(64,6)$ & $16(84,2)$ & $13(100,0)$ & $3(50,0)$ & 0,020 \\
\hline R. Gineco-Obs & $80(68,4)$ & $55(69,6)$ & $15(79,0)$ & $6(46,2)$ & $4(66,7)$ & 0,260 \\
\hline R. Cirugía & $70(59,8)$ & $49(62,0)$ & $7(36,8)$ & $10(76,9)$ & $4(66,7)$ & 0,110 \\
\hline R. Pediatría & $82(70,1)$ & $59(74,7)$ & $11(57,9)$ & $8(61,5)$ & $4(66,7)$ & 0,452 \\
\hline
\end{tabular}

* Diferencia significativa encontrada mediante la prueba de Kruskal-Wallis. Posteriormente se realizó la prueba para comparar cada par, encontrando diferencia significativa entre Clínicas y Essalud ( $p=0,0124)$, y entre Clinicas y MINSA ( $p=0,0205)$.

** Diferencia significativa encontrada mediante la prueba de Kruskal-Wallis. Posteriormente se realizó la prueba para comparar cada par, encontrando diferencia significativa entre Essalud y MINSA ( $p=0,0044)$.

p: Prueba de Kruskal-Wallis para variables numéricas, y prueba exacta de Fisher para variables categóricas; R. = rotación. 
des MINSA/EsSalud que en Clínicas $(\mathrm{p}=0,035)$. El promedio del número de guardias por mes fue $7,3 \pm 3,7$, siendo significativamente mayor en MINSA que en EsSalud ( $p=0,014)$. La frecuen- cia de carga laboral excesiva y de 'papeleo excesivo' en al menos una rotación fue de $80,3 \%$ y $84,6 \%$ respectivamente, siendo ambas variables mayores en sedes MINSA/EsSalud que en Clínicas $(\mathrm{p}=0,001)$. La frecuencia de docencia adecuada en todas las rotaciones fue de 30,8\%, siendo mayor en Sanidades y EsSalud para la rotación de Medicina $(p=0,020)($ tabla 2).

Tabla 3. Autopercepción de adecuada capacitación en la realización de procedimientos durante el internado médico en la población encuestada.

\begin{tabular}{|c|c|c|c|c|c|c|}
\hline Procedimientos & Total & $\begin{array}{l}\text { MINSA } \\
(\mathrm{N}=79)\end{array}$ & $\begin{array}{l}\text { EsSalud } \\
(\mathrm{N}=19)\end{array}$ & $\begin{array}{l}\text { Sanidades } \\
(\mathrm{N}=13)\end{array}$ & $\begin{array}{l}\text { Clínicas } \\
(\mathrm{N}=6)\end{array}$ & $\mathrm{p}$ \\
\hline \multicolumn{7}{|l|}{ Clínicos } \\
\hline Toma de presión arterial & $106(90,6)$ & $70(88,6)$ & $19(100,0)$ & $12(92,3)$ & $5(83,3)$ & 0,431 \\
\hline Tomar una muestra de sangre arterial & $99(84,6)$ & $70(88,6)$ & $17(89,5)$ & $8(61,5)$ & $4(66,7)$ & 0,044 \\
\hline Colocación de sonda Foley & $97(82,9)$ & $63(79,8)$ & $18(94,7)$ & $12(92,3)$ & $4(66,7)$ & 0,225 \\
\hline Toma de electrocardiograma & $89(76,1)$ & $56(70,9)$ & $18(94,7)$ & $11(84,6)$ & $4(66,7)$ & 0,132 \\
\hline Realizar RCP básico en pacientes adultos & $84(71,8)$ & $61(77,2)$ & $13(68,4)$ & $6(46,2)$ & $4(66,7)$ & 0,136 \\
\hline $\begin{array}{l}\text { Oxigenoterapia (administración de oxígeno a diversas } \\
\text { concentraciones) }\end{array}$ & $77(65,8)$ & $52(65,8)$ & $12(63,2)$ & $9(69,2)$ & $4(66,7)$ & 0,988 \\
\hline Interpretación de electrocardiograma & $72(61,5)$ & $46(58,2)$ & $13(68,4)$ & $9(69,2)$ & $4(66,7)$ & 0,768 \\
\hline Tomar una muestra de sangre venosa & $64(54,7)$ & $44(55,7)$ & $13(68,4)$ & $4(30,8)$ & $6(100,0)$ & 0,209 \\
\hline Colocación de inyectables (SC, IM, IV) & $63(53,9)$ & $46(58,2)$ & $10(52,6)$ & $5(38,5)$ & $2(33,3)$ & 0,411 \\
\hline Atención ambulatoria (consultorio) de dolencias comunes & $60(51,3)$ & $34(43,0)$ & $10(52,6)$ & $11(84,6)$ & $5(83,3)$ & 0,015 \\
\hline Realizar RCP básico en pacientes pediátricos & $58(49,6)$ & $40(53,6)$ & $8(42,1)$ & $6(46,2)$ & $4(66,7)$ & 0,748 \\
\hline Colocación de tubo endotraqueal & $53(45,3)$ & $37(46,8)$ & $7(36,8)$ & $5(38,5)$ & $4(66,7)$ & 0,578 \\
\hline Calcular y aplicar una infusión endovenosa & $49(41,9)$ & $33(41,8)$ & $10(52,6)$ & $4(30,8)$ & $2(33,3)$ & 0,628 \\
\hline Colocar un catéter venoso periférico & $43(36,8)$ & $30(38,0)$ & $8(42,1)$ & $3(23,1)$ & $2(33,3)$ & 0,715 \\
\hline Colocar un catéter venoso central & $33(28,2)$ & $23(29,11)$ & $7(36,8)$ & $0(0,0)$ & $3(50,0)$ & 0,064 \\
\hline Manejo farmacológico de pacientes psiquiátricos & $25(21,4)$ & $17(21,5)$ & $4(21,1)$ & $2(15,4)$ & $2(33,3)$ & 0,852 \\
\hline \multicolumn{7}{|l|}{ Quirúrgicos } \\
\hline Suturar piel & $106(90,6)$ & $70(88,6)$ & $19(100,0)$ & $12(92,3)$ & $5(83,3)$ & 0,431 \\
\hline Suturar piel y músculo & $97(82,9)$ & $66(83,5)$ & $17(89,5)$ & $9(69,2)$ & $5(83,3)$ & 0,509 \\
\hline Incisión y drenaje de abscesos superficiales & $82(70,1)$ & $53(67,1)$ & $17(89,5)$ & $7(53,9)$ & $5(83,3)$ & 0,117 \\
\hline $\begin{array}{l}\text { Aplicar primeros auxilios (ABCDE) en pacientes poli- } \\
\text { traumatizados }\end{array}$ & $52(65,8)$ & $8(61,6)$ & $10(52,6)$ & $8(61,5)$ & $5(83,3)$ & 0,534 \\
\hline Extraer una uña & $60(51,3)$ & $37(46,8)$ & $12(63,2)$ & $8(61,6)$ & $3(50,0)$ & 0,522 \\
\hline Inmovilización de fracturas cerradas & $51(43,6)$ & $38(48,1)$ & $5(26,3)$ & $2(15,4)$ & $6(100,0)$ & 0,002 \\
\hline Reducción de fracturas & $33(28,2)$ & $21(26,6)$ & $4(21,1)$ & $2(15,4)$ & $6(100,0)$ & 0,001 \\
\hline \multicolumn{7}{|l|}{ Ginecológicos - neonatales } \\
\hline Realizar atención inmediata del recién nacido & $114(97,4)$ & $78(98,7)$ & $17(89,5)$ & $13(100,0)$ & $6(100,0)$ & 0,119 \\
\hline Atender un parto vaginal & $106(90,6)$ & $72(91,1)$ & $17(89,5)$ & $12(92,3)$ & $5(83,3)$ & 0,925 \\
\hline Realizar un tacto vaginal & $103(88,0)$ & $69(87,3)$ & $17(89,5)$ & $13(100,0)$ & $4(66,7)$ & 0,218 \\
\hline $\begin{array}{l}\text { Manejo de emergencias obstétricas (preeclampsia, } \\
\text { hemorragia, sepsis, etc) }\end{array}$ & $101(86,3)$ & $70(88,6)$ & $17(89,5)$ & $10(76,9)$ & $4(66,7)$ & 0,328 \\
\hline Realizar un legrado endometrial & $69(59,0)$ & $42(53,2)$ & $14(73,7)$ & $10(76,9)$ & $3(50,0)$ & 0,193 \\
\hline Atender un parto vaginal vertical & $32(27,4)$ & $20(25,3)$ & $6(31,6)$ & $3(23,1)$ & $3(50,0)$ & 0,572 \\
\hline Número de procedimientos & & & & & & 0,420 \\
\hline Tercil 1 (4 a 16 procedimientos) & $41(25,0)$ & $28(35,4)$ & $5(26,3)$ & $6(46,1)$ & $2(33,3)$ & \\
\hline Tercil 2 (17 a 20 procedimientos) & $39(33,3)$ & $28(35,4)$ & $7(36,8)$ & $4(30,8)$ & $0(0,0)$ & \\
\hline Tercil 3 (21 a 29 procedimientos) & $37(31,6)$ & $23(29,1)$ & $7(36,8)$ & $3(23,1)$ & $4(66,6)$ & \\
\hline
\end{tabular}

$\mathrm{RCP}=$ reanimación cardiopulmonar. 
Se evaluó la capacitación percibida en 29 procedimientos médicos. Pocos encuestados refirieron haber recibido durante su internado una adecuada capacitación en ciertos procedimientos, como la colocación de inyectables $(53,9 \%)$, cálculo y aplicación de infusión endovenosa $(41,9 \%)$, colocación de catéter venoso periférico $(36,8 \%)$, inmovilización de fracturas cerradas $(43,6 \%)$, reducción de fracturas (28,2\%), y atención de partos vaginales verticales $(27,4 \%)$ (tabla 3$)$.

Al evaluar los factores asociados a una adecuada capacitación en más de 20 procedimientos, las siguientes variables resultaron significativas en el análisis multivariado: presentar una adecuada docencia en tres o cuatro rotaciones, en comparación con presentarla en dos o menos rotaciones $(\mathrm{RP}=2,55, \quad \mathrm{IC} 95 \%=1,36-4,76)$; y laborar el promedio de 9 horas y 30 minutos a 10 horas 15 minutos diarios en comparación con laborar de 6 a 9 horas y 15 minutos diarios $(\mathrm{RP}=1,91$, IC95\% $=1,01-3,64)$ (tabla 4).

\section{DISCUSIÓN}

Los encuestados percibieron no haber recibido una adecuada capacitación en algunos de los procedimientos médicos analizados. Existen dos posibles explicaciones para estos resultados: una explicación es que los estudiantes le hayan restado importancia a estos procedimientos durante el internado porque ya se sentían adecuadamente capacitados en estos; otra explicación es que los estudiantes nunca hayan sido capacitados en la realización de dichos procedimientos, y terminen su carrera sin las competencias necesarias para su correcta realización. Dado que en Perú el internado suele ser la etapa donde se consolidan casi todas las competencias de los estudiantes, nos inclinamos por la segunda opción.

En cuanto a los procedimientos clínicos, pocos encuestados percibieron una adecuada capacitación en colocación de inyectables, colocación de catéter venosos periféricos y cálculo y aplicación de infusiones endovenosas.

Tabla 4. Factores asociados a la percepción de adecuada capacitación en más de 20 procedimientos.

\begin{tabular}{|c|c|c|}
\hline & $\begin{array}{l}\text { Análisis crudo } \\
\text { RP (IC95\%) }\end{array}$ & $\begin{array}{l}\text { Análisis ajustado } \\
\text { RP (IC95\%) }\end{array}$ \\
\hline Edad de 26 o más años & $1,17(0,67$ a 2,04$)$ & $1,05(0,60$ a 1,84$)$ \\
\hline Sexo femenino & $0,82(0,48$ a 1,39$)$ & $0,81(0,48$ a 1,37$)$ \\
\hline $\begin{array}{l}\text { Presentar carga laboral excesiva en tres o cuatro } \\
\text { rotaciones }\end{array}$ & $0,78(0,43$ a 1,42$)$ & $0,66(0,38$ a 1,16$)$ \\
\hline $\begin{array}{l}\text { Presentar docencia adecuada en tres o cuatro } \\
\text { rotaciones }\end{array}$ & $2,58(1,33$ a 4,98$)$ & $2,55(1,36$ a 4,76$)$ \\
\hline Haber realizado el internado en un Hospital Nivel IV & $0,67(0,27$ a 1,66$)$ & $0,71(0,26$ a 1,93$)$ \\
\hline $\begin{array}{l}\text { Haber realizado el internado en un Hospital del } \\
\text { MINSA }\end{array}$ & $0,79(0,46$ a 1,36$)$ & $0,60(0,32$ a 1,12$)$ \\
\hline \multicolumn{3}{|l|}{ Horas laboradas al día en promedio } \\
\hline Tercil 1 (6:00 a 9:15 horas) & 1,00 & 1,00 \\
\hline Tercil 2 (9:30 a 10:15 horas) & $1,72(0,86$ a 3,45$)$ & $1,91(1,01$ a 3,64$)$ \\
\hline Tercil 3 (10:30 a 18:00 horas) & $1,70(0,86$ a 3,39$)$ & $1,64(0,83$ a 3,24$)$ \\
\hline \multicolumn{3}{|l|}{ Guardias al mes en promedio } \\
\hline Tercil 1 (2 a 5 guardias) & 1,00 & 1,00 \\
\hline Tercil 2 (6 a 8 guardias) & $1,50(0,76$ a 2,95$)$ & $1,30(0,67$ a 2,53$)$ \\
\hline Tercil 3 (9 a 18 guardias) & $1,41(0,69$ a 2,86$)$ & $1,61(0,76$ a 3,37$)$ \\
\hline
\end{tabular}

Para el análisis crudo y multivariado se utilizó la regresión de Poisson simple con varianzas robustas y la regresión de Poisson multivariada con varianzas robustas, respectivamente. Se excluyó la variable 'papeleo excesivo', debido a que presentó colinealidad con 'carga laboral excesiva'.

Esto coincide con otros reportes extranjeros ${ }^{(19)}$, y puede deberse a la tercerización de estas labores al personal de enfermería ${ }^{(20)}$. Sin embargo, si bien en un ambiente hospitalario es recomendable el manejo multidisciplinario que delega al personal de enfermería la realización de ciertos procedimientos ${ }^{(2,21)}$, cabe recalcar que en Perú es muy probable que el médico tenga que realizar el SERUMS en lugares donde no hay otro personal de salud calificado, por lo cual su falta de entrenamiento en estos procedimientos resulta desventajosa ${ }^{(22)}$.

En cuanto a los procedimientos quirúrgicos y gineco-obstétricos, la mayor parte de los encuestados percibió haber recibido durante su internado una adecuada capacitación en la realización de suturas, drenajes de abscesos, atención de partos y atención inmediata de recién nacidos. Esto refleja la gran participación que tienen los internos en estos procedimientos básicos, que van a requerir en el SERUMS. Sin embargo, pocos fueron capacitados en otros procedimientos como la inmovilización y reducción de fracturas. Esto puede deberse a que los internos no están rotando el tiempo necesario en la especialidad de Traumatología, que en algunas sedes estos procedimientos se realizan pocas veces, o que no se permite que los internos las realicen. Es importante que futuros estudios describan esta problemática y permitan implementar las soluciones pertinentes en los currículos de los médicos peruanos ${ }^{(23)}$.

Otro procedimiento con carencias de capacitación fue la atención de parto vaginal vertical, probablemente debido a que esta modalidad de atención de parto no suele darse en las sedes hospitalarias debido a la ausencia de capacitación y de infraestructura especializada, prefiriéndose el parto horizontal ${ }^{(24)}$. Esto resulta en un problema para el médico que realiza el SERUMS, que se ve enfrentado a un ambiente distinto, y muchas veces es obligado a atender partos verticales sin ninguna capacitación formal previa. 
En nuestros resultados, pertenecer al segundo tercil en horas laboradas estuvo asociado a percibir una capacitación adecuada en más de 20 procedimientos, en comparación al primer tercil. Esto puede deberse a que el estudiante con más horas laboradas: [1] tiene más oportunidades de presenciar y eventualmente realizar procedimientos, especialmente en emergencias, donde estas oportunidades dependen del azar, [2] tiene más oportunidades de ganarse la confianza de sus residentes o asistentes, suficiente como para solicitarles participar en la realización de procedimientos, y [3] los médicos que observan las largas jornadas laborales del interno pueden verse más dispuestos a enseñarle y dejarle realizar los procedimientos. Sin embargo, el tercer tercil ya no presentó asociación, posiblemente debido a que un horario extenuante afecta negativamente al interno, atentando contra el aprendizaje.

El horario de los internos de medicina ha sido históricamente un tema de intenso debate. Actualmente existen directivas al respecto que imponen un máximo de 80 horas semanales en Estados Unidos ${ }^{(25)}, 48$ en Europa ${ }^{(26)} \mathrm{y}$ 37,5 en España ${ }^{(27)}$. En el caso de Perú, la jornada del interno en medicina se rige por el Decreto supremo $N^{\circ} 003$ 2008 TR "que dicta medidas sobre jornadas máximas del [...] internado en Ciencias de la Salud", el cual establece una jornada laboral máxima en establecimientos de salud del Sector Público de 6 horas diarias, 36 horas semanales o 150 horas mensuales, incluyendo las guardias nocturnas; así como un periodo de descanso no menor de cinco horas, tanto previo como posterior a las guardias nocturnas. Sin embargo, estas normas rara vez son respetadas, ya sea por imposición de los médicos que tienen a cargo a los internos, o por voluntad propia de los internos ${ }^{(28)}$, como se refleja en nuestros resultados, donde se encontró que los internos laboraron en promedio $10,0 \pm 1,8$ horas diarias sin contar las guardias nocturnas.

El número de horas laboradas no es uniforme entre las sedes, y resulta mayor en las sedes de MINSA y EsSalud, lo que puede estar reflejando la dinámica que existe en muchas sedes hospitalarias, en las cuales el interno es visto como 'mano de obra barata' (o gratuita), que debe realizar largas jornadas laborales a cambio de la docencia recibida, siendo obligado a suplir las deficiencias de personal y recursos del hospital, lo cual generalmente implica tener que realizar grandes cantidades de trabajo administrativo y trámites documentarios. En casos extremos, el tiempo invertido en estas labores disminuye en gran magnitud el tiempo efectivo de aprendizaje (que debería incluir visitas médicas, realización de procedimientos, atenciones en emergencia, actividades académicas, asistencia a conferencias, lectura y reflexión), reduciendo al interno a ser un tramitador más ${ }^{(10)}$. Además, las jornadas agotadas están asociadas a un mayor número de errores médicos, lo cual puede repercutir en la salud de los pacientes ${ }^{(29)}$.

Por ello, resulta necesario establecer un límite en la jornada laboral de los internos, que sea cumplido por las sedes hospitalarias y que permita que estos alcancen las competencias requeridas. También es importante que se priorice la docencia del interno sobre su utilidad para el hospital, y no se recargue al interno con labores que no contribuyan a su formación.

Otro factor asociado a percibir una adecuada capacitación en más de 20 procedimientos fue haber recibido una docencia adecuada en al menos tres rotaciones. En la actualidad, la docencia que recibe el interno queda casi exclusivamente a cargo del personal de salud de la sede hospitalaria (en especial los médicos residentes o asistentes), y depende en buena parte de la habilidad docente de estos ${ }^{(30)}$. Por ello, una estrategia efectiva es implementar programas de entrenamiento en habilidades docentes a los residentes ${ }^{(31)}$, con el fin de mejorar la supervisión y el feedback que realizan a los internos ${ }^{(32)}$.

El aprendizaje de los procedimientos debe ser realizado en tres etapas suce- sivas: cognición (el estudiante entiende el procedimiento), integración (el estudiante realiza el procedimiento) y automatización (el estudiante realiza el procedimiento con velocidad, eficiencia y precisión), siendo las dos primeras etapas realizadas en ambientes simulados ${ }^{(6)}$. Por ello, es importante que se destine un tiempo a la capacitación y evaluación de ciertas habilidades en simuladores ${ }^{(40)}$, en especial para aquellos procedimientos que no se presenten frecuentemente y en los cuales probablemente ni siquiera los médicos de mayor rango sean expertos ${ }^{(33)}$.

Los simuladores se vienen usando cada vez con más frecuencia en la educación médica, y han demostrado ser útiles para desarrollar numerosas competencias y habilidades procedimentales ${ }^{(34-36)}$, incluso si se utilizan durante periodos cortos o en talleres previos al internado ${ }^{(37-39)}$. Obviamente esto representa la inversión económica, cuya procedencia deberá ser discutida en favor de la docencia hospitalaria no solo de internos sino de estudiantes y residentes, en los cuales también se han evidenciado deficiencias ${ }^{(41,42)}$.

Por último, cabe recalcar que el internado en Perú dura un año, a diferencia de otros países como Chile ${ }^{(43)}$ y México ${ }^{(3)}$ en los que dura dos años. Si bien las universidades tendrían dificultades para aumentar la duración del internado, una alternativa radica en la realización de un 'externado', referido a rotaciones previas al internado orientadas a maximizar la experiencia práctica del estudiante, que algunas universidades peruanas ya han adoptado ${ }^{(44)}$.

Una limitación importante para el presente estudio es la falta de una evaluación objetiva de los procedimientos médicos, lo cual limita los resultados a las expectativas y los recuerdos del interno. Además, se presentó una alta tasa de rechazo, siendo probable que aquellos que se perciban más capacitados hayan tenido mayor intención de participar que aquellos que no se sientan así, y que por tanto estemos sobreestimando la capacitación percibi- 
da en el internado. Otras limitaciones son la escasa cantidad de participantes que hayan realizado el internado en las Sanidades y en Clínicas, y el sesgo de memoria. Sin embargo, este es a nuestro conocimiento el primer estudio que evalúa la capacitación que perciben los internos de Medicina en el Perú, cuyos resultados nos brindan luces sobre la magnitud de esta problemática y los posibles factores asociados.

En conclusión, con respecto al internado médico se encontró que un alto porcentaje de los encuestados perciben que la carga laboral y el papeleo fueron excesivos en al menos una de sus rotaciones; y que no se les brindó una adecuada capacitación en varios de los procedimientos evaluados. Además, percibirse adecuadamente capacitado en más de 20 procedimientos estuvo asociado al promedio del número de horas laboradas al día, y a percibir una docencia adecuada en al menos tres rotaciones. Es necesario que futuros estudios regulen la jornada laboral, el rol de los internos y las actividades docentes que se realizan.

\section{REFERENCIAS BILBLIOGRÁFICAS}

1. Asociación Peruana de Facultades de Medicina. Reglamento del comité nacional de pregrado de salud del SINAPRES. ASPEFAM; 2006.

2. Duffy FD, Holmboe ES. What procedures should internists do? Ann Intern Med. 6 de marzo de 2007;146(5):392-3. doi:10.7326/0003-4819-1465-200703060-00012.

3. Tapia Villanueva RM, Núñez Tapia RM, Syr Salas Perea R, Rodriguez-Orozco AR. El internado médico de pregrado y las competencias clínicas: México en el contexto latinoamericano. Educ Médica Super. Diciembre de 2007;21(4):0-0.

4. Inga-Berrospi F, Taype-Rondán Á, Purizaca-Rosillo N. La problemática del médico serumista en el Perú: conclusiones de la Segunda Convención Nacional de Médicos Serumistas, 2013. An Fac med. Julio de 2014;75(3):271-2. DOI: http://dx.doi. org/10.15381/anales.v75i3.9785.

5. Mayta-Tristán P, Poterico JA, Galán-Rodas E, RaaOrtiz D. El requisito obligatorio del servicio social en salud del Perú: discriminatorio e inconstitucional. Rev Peru Med Exp Salud Publica. Octubre de 2014;31(4):781-7.

6. Reznick RK, MacRae H. Teaching surgical skills - Changes in the wind. N Engl J Med. 21 de diciembre de 2006;355(25):2664-9. 2006DOI: 10.1056/NEJMra054785.

7. Luna-Porta L, Mayor-Vega A, Taype-Rondán Á. Sindrome de burnout en estudiantes de pregrado de medicina humana: un problema escasamente estudiado en el Perú. An Fac med. Enero de 2015;76(1):83-4. DOI: http://dx.doi.org/10.15381/ anales.v76i1.11082.

8. Shea JA, Bellini LM, Dinges DF, Curtis ML, Tao Y Zhu J, Small DS, Basner M, Norton L, Novak C, Dine CJ, Rosen IM, Volpp KG. Impact of protected sleep period for internal medicine interns on overnight call on depression, burnout, and empathy. J Grad Med Educ. 2 de abril de 2014;6(2):256-63. doi: 10.4300/ JGME-D-13-00241.1.

9. Christino MA, Matson AP, Fischer SA, Reinert SE Digiovanni CW, Fadale PD. Paperwork versus patient care: a nationwide survey of residents' perceptions of clinical documentation requirements and patient care. J Grad Med Educ. 15 de octubre de 2013;5(4):600-4. doi: 10.4300/JGMED-12-00377.1.

10. Siegler JE, Patel NN, Dine CJ. Prioritizing paperwork over patient care: why can't we do both? J Grad Med Educ. 2015;7(1):16-8. doi: http://dx.doi. org/10.4300/JGME-D-14-00494.1.

11. Block L, Habicht R, Wu AW, Desai SV, Wang K, Silva KN, et al. In the wake of the 2003 and 2011 duty hours regulations, how do internal medicine interns spend their time? J Gen Intern Med. 18 de abril de 2013;28(8):1042-7. doi: 10.1007/s11606013-2376-6.

12. Comisión para la Acreditación de Facultades o Escuelas de Medicina. Ley, Normas de Ejecución y Estándares Mínimos para la Acreditación de Facultades o Escuelas de Medicina. Lima: CADME; 2001.

13. Simpson JG, Furnace J, Crosby J, Cumming $A D$, Evans PA, Friedman Ben David M, et al. The Scottish doctor--learning outcomes for the medical undergraduate in Scotland: a foundation for competent and reflective practitioners. Med Teach. Marzo de 2002;24(2):136-43.

14. Tomorrow's Doctors: Outcomes 2 - The doctor as a practitioner [Internet]. [citado 30 de julio de 2015]. Recuperado a partir de: http://www.gmc-uk. org/education/undergraduate/tomorrows_doctors_2009_outcomes2.asp

15. 15. Englander R, Cameron T, Ballard A, Dodge J, Bull J, Aschenbrener C. Toward a common taxonomy of competency domains for the health professions and competencies for physicians Academic Medicine. 2013;88(8):1088-94. doi: 10.1097/ACM.0b013e31829a3b2b.

16. Proyecto Tuning para América Latina. Competencias Específicas de Medicina [Internet]. [citado 30 de julio de 2015]. Recuperado a partir de: http:// tuning.unideusto.org/tuningal/index.php?option $=\mathrm{C}$ ontent\&task=view\&id=229\&/temid $=258$

17. Jason RF. The CanMEDS 2005 Physician Competency Framework - Better standards. Better physicians. Better care. Canada: The CanMEDS; 2005.

18. Ministerio de Salud del Perú. Categorías de establecimientos del Sector Salud. N T N.o 0021 MINSA / DGSP V.01. Lima: Dirección General de Salud de las Personas, MINSA; 2004.

19. Coberly L, Goldenhar LM. Ready or not, here they come: acting interns' experience and perceived competency performing basic medical procedures. J Gen Intern Med. Abril de 2007;22(4):491-4. doi: 10.1007/s11606-007-0107-6.

20. Horrocks S, Anderson E, Salisbury C. Systematic review of whether nurse practitioners working in primary care can provide equivalent care to doctors. BMJ. 6 de abril de 2002;324(7341):819-23. doi: http://dx.doi.org/10.1136/bmj.324.7341.819.

21. Vazirani S, Hays RD, Shapiro MF, Cowan M. Effect of a multidisciplinary intervention on communi- cation and collaboration among physicians and nurses. Am J Crit Care Off Publ Am Assoc Crit-Care Nurses. Enero de 2005;14(1):71-7.

22. Taype-Rondán A, Inga-Berrospi F, Casiano Celestino R, Bastidas F. Percepción de médicos egresados sobre las habilidades clínicas adquiridas durante el pregrado en Lima. Rev Med Chile 2015;143:540-2.

23. Bellamy N, Goldstein LD, Tekanoff RA, Support, Non-U.S.Gov't. Continuing medical educationdriven skills acquisition and impact on improved patient outcomes in family practice setting. J Contin Educ Health Prof. 2000;20(1):52-61.

24. Nureña CR. Incorporación del enfoque intercultural en el sistema de salud peruano: la atención del parto vertical. Rev Panam Salud Publica. 2009;26(4):368-76. Doi: http://dx.doi.org/10.1590/ S1020-49892009001000013.

25. AAMC policy guidance on graduate medical education: assuring quality patient care and quality education. Washington, D.C.: Association of American Medical Colleges (AAMC); 2001.

26. Pickersgill T. The European working time directive for doctors in training. BMJ. 1 de diciembre de 2001;323(7324):1266. doi: http://dx.doi. org/10.1136/bmj.323.7324.1266.

27. España. Real Decreto $1146 / 2006$, de 6 de octubre, por el que se regula la relación laboral especial de residencia para la formación de especialistas en Ciencias de la Salud. Boletín Oficial del Estado; 2006.

28. Lee DY, Myers EA, Rehmani SS, Wexelman BA, Ross RE, Belsley SS, et al. Surgical residents' perception of the 16-hour work day restriction: concern for negative impact on resident education and patient care. J Am Coll Surg. Diciembre de 2012;215(6):868-77. doi: 10.1016/j.jamcollsurg.2012.08.005.

29. Landrigan CP, Rothschild JM, Cronin JW, Kaushal R, Burdick E, Katz JT, et al. Effect of Reducing Interns' Work Hours on Serious Medical Errors in Intensive Care Units. N Engl J Med. 28 de octubre de 2004;351(18):1838-48.

30. Arah OA, Heineman MJ, Lombarts KMJMH. Factors influencing residents' evaluations of clinical faculty member teaching qualities and role model status. Med Educ. Abril de 2012;46(4):381-9. doi: 10.1111/j.1365-2923.2011.04176.x.

31. Morrison E, Friedland JA, Boker J, Rucker L, Hollingshead J, Murata P. Residents-as-teachers Training in U.S. Residency Programs and offices of graduate medical education. Academic Medicine. Octubre 2011;76(10):S1-4.

32. Smith CC, Gordon CE, Feller-Kopman D, Huang GC, Weingart SN, Davis RB, et al. Creation of an innovative inpatient medical procedure service and a method to evaluate house staff competency. J Gen Intern Med. Mayo de 2004;19(5 Pt 2):510-3.

33. Wickstrom GC, Kelley DK, Keyserling TC, Kolar MM, Dixon JG, Xie SX, et al. Confidence of academic general internists and family physicians to teach ambulatory procedures. J Gen Intern Med. Junio de 2000;15(6):353-60.

34. Graafland M, Schraagen JM, Schijven MP. Systematic review of serious games for medical education and surgical skills training. $\mathrm{Br} \mathrm{J}$ Surg. Octubre de 2012;99(10):1322-30. doi: 10.1002/bjs.8819.

35. McGaghie WC, Issenberg SB, Cohen ER, Barsuk JH, Wayne DB. Does simulation-based medical education with deliberate practice yield better results than traditional clinical education? A meta-analytic comparative review of the evidence. Acad Med J Assoc Am Med 
Coll. Junio de 2011;86(6):706-11. doi: 10.1097/ ACM.0b013e318217e119.

36. Schroedl CJ, Corbridge TC, Cohen ER, Fakhran SS, Schimmel D, McGaghie WC, et al. Use of simulation-based education to improve resident learning and patient care in the medical intensive care unit: a randomized trial. J Crit Care. Abril de 2012;27(2):219.e7-13. doi: 10.1016/j. jcrc.2011.08.006.

37. Cohen ER, Barsuk JH, Moazed F, Caprio T, Didwania A, McGaghie WC, et al. Making July safer: simulation-based mastery learning during intern boot camp. Acad Med J Assoc Am Med Coll. Febrero de 2013;88(2):233-9. doi: 10.1097/ ACM.0b013e31827bfc0a

38. Peyre SE, Peyre CG, Sullivan ME, Towfigh S. A surgical skills elective can improve student confidence prior to internship. J Surg Res. Junio de 2006;133(1):11-5.

39. Teo AR, Harleman E, O'sullivan PS, Maa J. The key role of a transition course in preparing medical students for internship. Acad Med J Assoc Am Med Coll. Julio de 2011;86(7):860-5. doi: 10.1097/ ACM.0b013e31821d6ae2.

40. Champin D. Evaluación por competencias en la educación médica. Rev Peru Med Exp Salud Publica. Julio de 2014;31(3):566-71.

41. Herrera-Añazco P, Hernández AV, Sánchez-Rivas F, Arana Maestre C. Percepción de la formación durante el residentado médico en hospitales del Ministerio de Salud de Lima, Perú. Rev Peru Med Exp Salud Publica. Julio de 2014;31(3):606-7.

42. Miní E, Medina J, Peralta V, Rojas L, Butron J, Gutiérrez EL. Programa de residentado médico: percepciones de los médicos residentes en hospitales de Lima y Callao. Rev Peru Med Exp Salud Pública. Junio de 2015;32(2):303-10.

43. Millán K. T, Vargas C. NA, Madrid C. N. Internado rural en la carrera de medicina de la Universidad de Chile; una experiencia de aprendizaje significativo. Educ Médica. Septiembre de 2006;9(3):20-1. 44. Vásquez-Sullca RR, Saco-Méndez S, Pereira-
Victorio CJ. El internado rural como acercamiento a la atención primaria en salud, la experiencia de una universidad de Cusco, Perú. Rev Peru Med Exp Salud Pública. 2013;30(2):344-64.

Artículo recibido el 3 de octubre de 2015 y aceptado para publicación el 9 de diciembre de 2015.

Conflicto de intereses: No existen.

\section{Correspondencia:}

Alvaro Taype Rondán

Dirección: Calle Pietro Torrigiano, Num 159, Dpto 102, San Borja, Lima, Perú

Correo electrónico: alvaro_athos@hotmail.com

Teléfonos: $970001517 /(01) 2265586$ 